\title{
Endotracheal tube intubation with the aid of a laryngeal mask airway, a fiberoptic bronchoscope, and a tube exchanger in a difficult airway patient
} -a case report-

\author{
Joon Kyung Sung ${ }^{1}$, Hyung Gon Kim², Jung Eun Kim, Myung-Soo Jang ${ }^{2}$, and Jong-Man Kang ${ }^{3}$ \\ Department of Anesthesiology and Pain Medicine, ${ }^{1}$ Graduate School, Kyung Hee Medical University, ${ }^{2}$ Kyung Hee University Hospital, \\ ${ }^{3}$ Kyung Hee University Hospital at Gangdong, Seoul, Korea
}

A 28-year-old male patient with occipito-atlanto-axial instability underwent a cervical fusion with posterior technique. Post-operatively, the endotracheal tube (ETT) was removed, and the patient was transferred to the intensive care unit. After transfer, an upper airway obstruction developed and reintubations with a laryngoscope were attempted but failed. We inserted a \#4 proseal laryngeal mask airway (LMA) and passed a $5.0 \mathrm{~mm}$ ETT through the LMA with the aid of a fiberoptic bronchoscope. We passed a tube exchanger through the $5.0 \mathrm{~mm}$ ETT and exchanged it with a $7.5 \mathrm{~mm}$ ETT. This method may be a useful alternative for difficult tracheal intubations. (Korean J Anesthesiol 2014; 66: 237-239)

Key Words: Airway obstruction, Bronchoscopes, Cervical vertebrae, Laryngeal masks, Spinal fusion, Tube exchanger.

One of the potentially life-threatening complications of cervical spinal surgery is upper airway obstruction, which is not uncommon. This risk is especially great during the immediate post-operative period. Once airway obstruction occurs and reintubation is required, the involved practitioner must expect and be prepared for a difficult intubation. In our case, acute airway obstruction occurred immediately after tracheal extubation. We discuss the possible causes and the appropriate management in such circumstances with a thorough literature review. We report this case with written permission of the patient and our Institutional Review Board.

\section{Case Report}

A 28-year-old male patient diagnosed with occipito-atlantoaxial instability was scheduled for a cervical fusion. He was 76 $\mathrm{kg}, 169 \mathrm{~cm}$, Mallampati class III, with a thyromental distance of $7.5 \mathrm{~cm}$ and limited head and neck mobility. He was intubated with a fiberoptic bronchoscope (Olympus LF-GP, Olympus Optical Co., Tokyo, Japan) in an awake state, and general anesthesia was maintained with sevoflurane. The operation was performed via the posterior approach in the prone position. Total operation time was about 6 hours, and there were no significant intraoper-

Received: February 1, 2013. Revised: March 4, 2013. Accepted: March 8, 2013.

Corresponding author: Jong-Man Kang, M.D., Ph.D., Department of Anesthesiology and Pain Medicine, Kyung Hee University Hospital at Gangdong, 149, Sangil-dong Gangdong-gu, Seoul 134-090, Korea. Tel: 82-2-440-6193, Fax: 82-2-440-7808, E-mail: jongmankang@gmail.com (c) This is an open-access article distributed under the terms of the Creative Commons Attribution Non-Commercial License (http:// creativecommons.org/licenses/by-nc/3.0/), which permits unrestricted non-commercial use, distribution, and reproduction in any medium, provided the original work is properly cited. 
ative events. During emergence, after confirming his stable condition (clear consciousness, adequate weaning profile), he was extubated in the operating room and sent to the intensive care unit. However, immediate $\mathrm{O}_{2}$ desaturation with dyspnea was noted. After giving the patient $5 \mathrm{mg}$ of midazolam for sedation, reintubation with a laryngoscope was attempted twice but failed due to the short jaw-chest distance, neck rigidity, and the flexed cervical angle after cervical spine fixation. His Cormack and Lehane grade was 4 . The laryngoscopic view did not improve, even with adequate repositioning of the head (BURP maneuver: backward, upward, and right lateral displacement of the thyroid cartilage). Oxygen supply was continuously provided through a face mask to maintain $\mathrm{O}_{2}$ saturation above $90 \%$. Nasotracheal intubation with a fiberoptic bronchoscope was attempted but also failed due to severe swelling of the tongue, epiglottis, retropharyngeal soft tissue, and the upper airway. Another interfering factor was the bloody secretions in the oropharynx, which obscured the tip of the fiberoptic bronchoscope. As oxygen saturation was able to be maintained at $90 \%$ using bag-valve-mask ventilation and the patient was not at increased risk of aspiration of gastric contents, we decided to perform tracheal intubation via an appropriately sized laryngeal mask airway (LMA). Because there was no intubating laryngeal mask airway (ILMA) available in our facility, we used a proseal LMA as an alternative. A \#4 LMA was easily inserted and functioned properly. We passed a $5.0 \mathrm{~mm}$ endotracheal tube (ETT, without a balloon) through the LMA with the aid of a fiberoptic bronchoscope into the trachea. We then passed a tube exchanger with an outer diameter $3.7 \mathrm{~mm}$ (Cook Critical Care, Bloomington, Indiana, USA) through the $5.0 \mathrm{~mm}$ ETT in order to exchange the $5.0 \mathrm{~mm}$ ETT and LMA with a $7.5 \mathrm{~mm}$ ETT (Fig. 1). After successfully

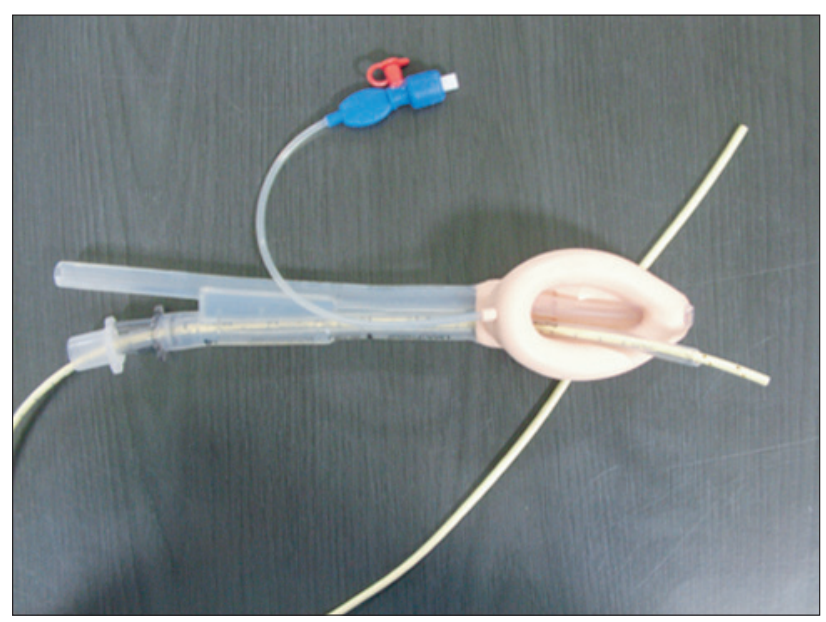

Fig. 1. A $5.0 \mathrm{~mm}$ endotracheal tube (without a balloon) passed through the proseal laryngeal mask airway with a tube exchanger inserted within the endotracheal tube. securing the airway, the patient was sedated with continuous infusion of midazolam and was mechanically ventilated overnight. The next day, extubation was carefully carried out by the anesthesiologist after examining cervical lateral radiographs for any signs of soft tissue edema. No further airway complications developed.

\section{Discussion}

Upper airway obstruction is a known life-threatening complication of cervical spinal surgery. Despite the low immediate reintubation rate (1.1-1.7\%), this complication can be critical once it develops [1]. The major known causes of airway obstruction after cervical spinal surgery are prevertebral soft tissue swelling [2], hematoma [3], cerebral fluid leakage [4], over-flexion fixation of the cervical spine [5], severe angioedema [6], and recurrent laryngeal nerve injury [7].

Several extubation strategies have been suggested in order to avoid respiratory complications after cervical spinal surgery. Epstein et al. [8] reported that intubation should be maintained for 24 hours and that by performing direct fiberoptic visualization of the degree of reactive tracheal swelling prior to extubation, airway complications might be reduced. In addition, extubation might be better performed with a tube exchanger, which can be removed in 5-10 minutes should oxygenation be adequate. In another clinical study, Suk et al. [9] suggested that high-risk patients should remain intubated for 2-3 days instead of only overnight.

Difficult airway is defined as the clinical situation in which a conventionally trained anesthesiologist experiences difficulty with face mask ventilation of the upper airway, difficulty with tracheal intubation, or both. Due to the limited range of motion of the cervical spine after surgical fusion and fixation in cervical spinal surgery, a difficult airway can be expected, and reintubation should be planned well and cautiously executed [1]. Fiberoptic intubation under spontaneous ventilation would be the first choice, considering that laryngoscopic intubation may be difficult and may possibly worsen the reactive changes of soft tissue. In case of a difficult intubation, a laryngeal mask could be used for maintaining a patent airway in such an emergency situation.

The laryngeal mask airway is an important option within the ASA difficult airway algorithm, and it has been proven to be a highly useful aid to fiberoptic intubation with ETT $[10,11]$. However, due to its construction, length, and diameter, there are limitations on the ETTs that can be passed through it [10]. The ILMA allows for easy passage of a well-lubricated $8.0-\mathrm{mm}$ internal diameter (ID) cuffed ETT, and it optimizes the angle at which the trachea is intubated. However, the ILMA has several disadvantages that become apparent with use: because of its cost, the ILMA may not always be available at every anesthesia work- 
station, and there are concerns about using the ILMA in patients with limited mouth opening [12,13] and in patients wearing semi-rigid neck collars [14]. Additionally, the ETT designed for use with the ILMA has a low volume/high pressure cuff, and anesthesiologists are reluctant to use these ETTs for prolonged periods [15]. They should be replaced as soon as feasible with a high volume/low pressure cuffed ETT, but this maneuver increases the risk of compromising the airway, especially in a patient with a difficult airway, and should be avoided if there is an alternative.

Some of the disadvantages of using LMA as an aid to intubate an ETT are the possibility of ETT misplacement along the vocal cord due to its short length, the limitation of the size of the ETT according to LMA size, and the difficulty in removing LMA after intubation [10]. Despite these disadvantages, in an environment where proseal LMA is the only option, our method may be of great use.

Overall, our case demonstrates an effective method of securing the airway in a difficult intubation case using a Proseal LMA, a fiberoptic bronchoscope, and a tube exchanger as an aid in performing intubation with ETT in circumstances where ILMA is not available or is better avoided. Length and diameter limitations on the ETTs that can be passed through the LMA can be overcome by using a tube exchanger as described in this case report.

\section{References}

1. Calder I, Calder J, Crockard HA. Difficult direct laryngoscopy in patients with cervical spine disease. Anaesthesia 1995; 50: 756-63.

2. Meakem TD, Meakem TJ, Rappaport W. Airway compromise from prevertebral soft tissue swelling during placement of halo-traction for cervical spine injury. Anesthesiology 1990; 73: 775-6.

3. Roy SP. Acute postoperative neck hematoma. Am J Emerg Med 1999; 17: 308-9.

4. Chang HS, Kondo S, Mizuno J, Nakagawa H. Airway obstruction caused by cerebrospinal fluid leakage after anterior cervical spine surgery. A report of two cases. J Bone Joint Surg Am 2004; 86-A: 370-2.

5. Harrop JS, Vaccaro A, Przybylski GJ. Acute respiratory compromise associated with flexed cervical traction after C2 fractures. Spine (Phila Pa 1976) 2001; 26: E50-4.

6. Krnacik MJ, Heggeness MH. Severe angioedema causing airway obstruction after anterior cervical surgery. Spine (Phila Pa 1976) 1997; 22: 2188-90.

7. Wattenmaker I, Concepcion M, Hibberd P, Lipson S. Upper-airway obstruction and perioperative management of the airway in patients managed with posterior operations on the cervical spine for rheumatoid arthritis. J Bone Joint Surg Am 1994; 76: 360-5.

8. Epstein NE, Hollingsworth R, Nardi D, Singer J. Can airway complications following multilevel anterior cervical surgery be avoided? J Neurosurg 2001; 94(2 Suppl): 185-8.

9. Suk KS, Kim KT, Lee SH, Park SW. Prevertebral soft tissue swelling after anterior cervical discectomy and fusion with plate fixation. Int Orthop 2006; 30: 290-4.

10. Benumof JL. Laryngeal mask airway and the ASA difficult airway algorithm. Anesthesiology 1996; 84: 686-99.

11. Kim JE, Chang CH, Nam YT. Intubation through a laryngeal mask airway by fiberoptic bronchoscope in an infant with a massat the base of the tongue. Korean J Anesthesiol 2008; 54: S43-6.

12. Asai T, Matsumoto H, Shingu K. Awake tracheal intubation through the intubating laryngeal mask. Can J Anaesth 1999; $46: 182-4$.

13. Brimacombe J, Keller C, Weidmann K. Limited mouth opening and the intubating laryngeal mask. Can J Anaesth 1999; 46: 807-8.

14. Wakeling HG, Nightingale J. The intubating laryngeal mask airway does not facilitate tracheal intubation in the presence of a neck collar in simulated trauma. Br J Anaesth 2000; 84: 254-6.

15. Honeybourne D, Costello JC, Barham C. Tracheal damage after endotracheal intubation: comparison of two types of endotracheal tubes. Thorax 1982; 37: 500-2. 